\title{
Cellular Learning Automata based Evolutionary Computing (CLA-EC) for Intrinsic Hardware Evolution
}

\author{
A. Hariri* R. Rastegar** K. Navi* M. Saheb Zamani** M. R. Meybodi** \\ arash hariri@itrc.ac.ir rrastegar@ce.aut.ac.ir navi@sbu.ac.ir szamani@ce.aut.ac.ir meybodi@ce.aut.ac.ir \\ * Shahid Beheshti University, Iran \\ **Amirkabir University of Technology, Iran
}

\begin{abstract}
Evolvable Hardware (EHW) deals with the application of evolutionary algorithms in hardware design. In intrinsic EHW, the evolutionary algorithm or the fitness evaluation is implemented in hardware. In this case, there is a need for hardware-friendly algorithms. In this work, we introduce Cellular Learning Automata based Evolutionary Computing (CLA-EC) as a new algorithm for intrinsic hardware evolution. The parallel structure of CLA-EC makes it suitable for EHW. Therefore, in this work we consider the application of this algorithm to EHW.
\end{abstract}

\section{Introduction}

Evolvable Hardware (EHW) is a new concept that targets the application of evolutionary algorithms to hardware design. The evolutionary Algorithms are used to solve optimization problem based on the concepts of natural evolution. Several evolutionary algorithms have been used in EHW and the most important algorithms are Genetic Programming (GP), Genetic Algorithms (GA), Evolutionary Strategies (ES), Compact Genetic Algorithms and Particle Swarm. In utilization of evolutionary algorithms in EHW two major methods have been introduced that are Intrinsic and extrinsic evolution. In extrinsic method, the optimization process of the used evolutionary algorithm and the fitness evaluation is carried out in software and after the optimization process, the optimum solution is implemented in hardware. In intrinsic method the fitness evaluation is carried out in hardware and also in some cases whole the algorithm is implemented in hardware. In the case, hardware imposes some restrictions to the implementation of evolutionary algorithms. The selection of the proper algorithms essentially affects the performance of the design. Intrinsic EHW requires some hardware-friendly evolutionary algorithms that can benefit the hardware implementation. Parallel nature of the algorithm, hardware utilization and power consumption are some of the properties that should be considered in choosing an evolutionary algorithm.

One of the most important benefits of hardware implementation is the consequent speed-up of the optimization process. Algorithms with parallel structure are the best candidates to be implemented in hardware. Some hardware based evolutionary algorithms have been previously developed [8][9] and also used for intrinsic EHW [10].

In this work, we introduce a new evolutionary algorithm for intrinsic EHW that is based on the parallel structure of the Cellular Automata (CA). Cellular Learning Automata based Evolutionary Computing (CLA-EC) is a parallel algorithm that benefits the properties of Cellular Learning Automata (CLA) [6][7] and Evolutionary Algorithms. CLA-EC has been introduced in [2] and has been successfully used in optimization applications and shown a good performance [2][4][5]. As will be discussed in the next section, CLA-EC can be efficiently implemented in digital hardware based on SIMD architecture. This parallel structure of CLA-EC makes it a good candidate for EHW.

In Section 2 we consider the concepts of Learning Automata, Cellular Learning Automata and CLA-EC then in Section 3 we evaluate the performance of the CLA-EC and consider its hardware architecture, and finally we conclude in Section 4.

\section{CLA-EC}

Learning Automata [1] are adaptive decisionmaking devices that operate in unknown random environments. A learning automaton has a finite set of actions and each action has a certain probability (unknown for the automaton) for getting rewarded by its environment. The aim is to learn to choose the optimal action (i.e. the action with the highest probability to be rewarded) through repeated interactions with the environment.

A Variable Structure Learning Automata (VSLA) model [1] is a 4-tuple $<\alpha, \beta, p, T(\alpha, \beta, p)>$, where $\alpha$ is 
a set of $s$ actions, $\beta$ is the environment response set and $p$ is a set that contains $s$ probabilities that each one is associated with the probability of performing one action in the current internal automaton state. $T$ is the reinforcement algorithm, which modifies the action probability, vector $p$, respecting the performed action and received response. Let a VSLA operate in an environment with $\beta=\{0,1\}$. Let $N$ be the set of nonnegative integers and the action $i$ be performed at instance $n \in N$. A general linear schema for updating action probabilities can be represented as follows.

If $\beta(n)=0$ (reward),

$$
\begin{gathered}
p_{i}(n+1)=p_{i}(n)+a\left[1-p_{i}(n)\right] \\
p_{j \neq i}(n+1)=(1-a) p_{j}(n) \\
\text { If } \beta(n)=1 \text { (penalty), } \\
p_{i}(n+1)=(1-b) p_{i}(n) \\
p_{j \neq i}(n+1)=(b / s-1)+(1-b) p_{j}(n)
\end{gathered}
$$

where $a$ and $b$ are reward and penalty parameters.

A Cellular Learning Automata model (CLA) [6][7] is a cellular automata [3] model in which a learning automaton (or multiple learning automata) is assigned to each cell. The learning automaton residing in a cell uses its action probabilities and determines the state (action) of that cell.

The CLA-EC [2] is a model obtained by combining the concepts of CLA and evolutionary algorithms. Like CLA, the CLA-EC involves some cells that form the population and each cell has a string genome. A set of learning automata (LA) is assigned to each cell and the set of actions selected by the LA, determines the string genome for that cell. Based on a local rule, a reinforcement signal vector is generated and given to the LA residing in the cell. Based on the received signal, each learning automaton updates its internal structure according to a learning algorithm. The process of action selection and updating the internal structure is repeated until a predetermined criterion is met. In the CLA-EC the cells are located on the nodes of a one-dimensional array with a wraparound connection and the interactions between the cells are restricted to the nearest neighbors.

The following steps will be repeated until a termination criterion is met.

1-Every automaton in cell $i$ chooses one of its actions using its action probability vector.

2-Every Cell $i$ generates a new string genome, new ${ }^{i}$ that is obtained by concatenating the selected actions ( 0 or 1 ) of the automata residing in that cell.

3 - Every cell $i$ computes the fitness value of new string genome $n e w^{i}$; if the fitness of this string genome is better than the one in the cell then the new string genome $n e w^{i}$ becomes the string genome of that cell. That is,

$$
\xi_{n+1}^{i}=\left\{\begin{array}{lr}
\xi_{n}^{i} & f\left(\xi_{n}^{i}\right) \leq f\left(n e w_{n+1}^{i}\right) \\
n e w_{n+1}^{i} & f\left(\xi_{n}^{i}\right)>f\left(n e w_{n+1}^{i}\right)
\end{array}\right.
$$

4- Every cell $i$ on the basis of the selected cells, generates a reinforcement vector and uses it as the input to its associated learning automata. Let $N(i)$ be the set of neighbors of cell $i$. Define,

where,

$$
N_{i, j}(k)=\sum_{l \in N(i)} \delta\left(\xi_{n}^{l, j}=k\right),
$$

$$
\delta(\exp )=\left\{\begin{array}{l}
1 \text { if } \exp \text { is true } \\
0 \text { otherwise. }
\end{array}\right.
$$

$\beta^{i, j}$, the reinforcement signal given to learning automaton $j$ of cell $i$, is computed as follows,

$$
\beta_{n}^{i, j}=\left\{\begin{array}{ll}
u\left(N_{i, j}(1)-N_{i, j}(0)\right) & \text { if } \xi_{n}^{i, j}=0 \\
u\left(N_{i, j}(0)-N_{i, j}(1)\right) & \text { if } \xi_{n}^{i, j}=1
\end{array},\right.
$$

where $u($.$) is a step function.$

Figure 1 shows the structure the CLA-EC and the communication of the cells. The internal structure of the cells has been depicted in Figure 2. The LA work in parallel to generate a new string genome. String genome determiner evaluates the string genome and the new string genome and chooses the better one as the current string genome.

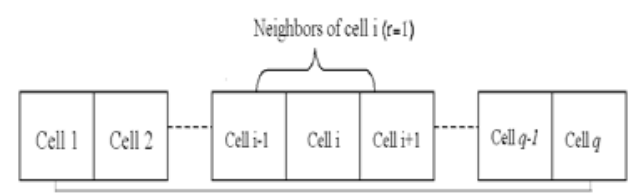

Figure 1. A one-dimensional (linear) CLA-EC

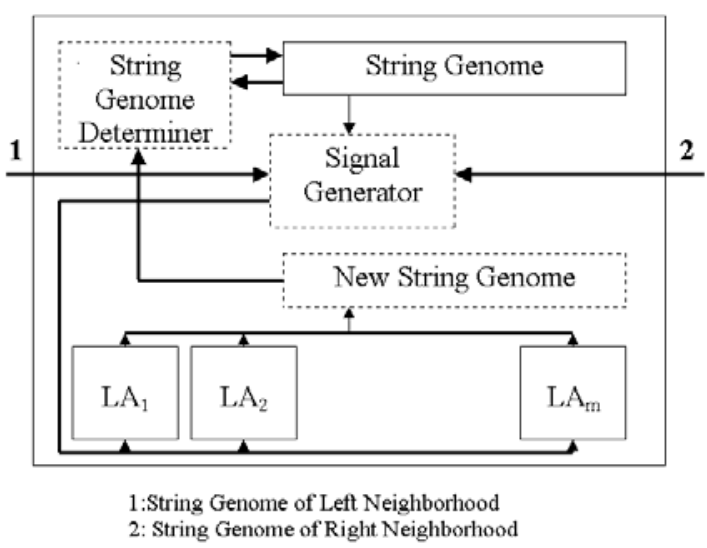

Figure 2. The structure of a cell 


\section{Experiments and Hardware Architecture}

To evaluate the performance of the CLA-EC, different problems and benchmarks have been approached that include DeJong's functions [11], OneMax problem and some useful applications. Training a neural network using the CLA-EC is one of these applications. To do that, CLA-EC has been used as a substitute for back-propagation algorithm for training multi layer neural networks. Result of using CLA-EC for approximating the function $y=\sin (x)$ has been depicted in Figure 3. The figure shows the convergence of the algorithm that is based on the average of five different runs.

As mentioned in Section 2, CLA-EC is composed of a number of cells, working in parallel to perform an optimization task. Each of the cells has its own string genome and computes the fitness value itself. Considering this structure of the algorithm, we design SIMD architecture for CLA-EC. In this architecture, the cells are the processing elements that are located in a ring topology according to the local communication among the cells. Figure 4 shows the topology of the SIMD architecture for this algorithm. This SIMD architecture creates a parallel system that achieves a high degree of parallelism that is suitable for intrinsic hardware evolution. In addition to this architecture, parallel architectures can be designed for the cells including their set of LA and fitness evaluation units.

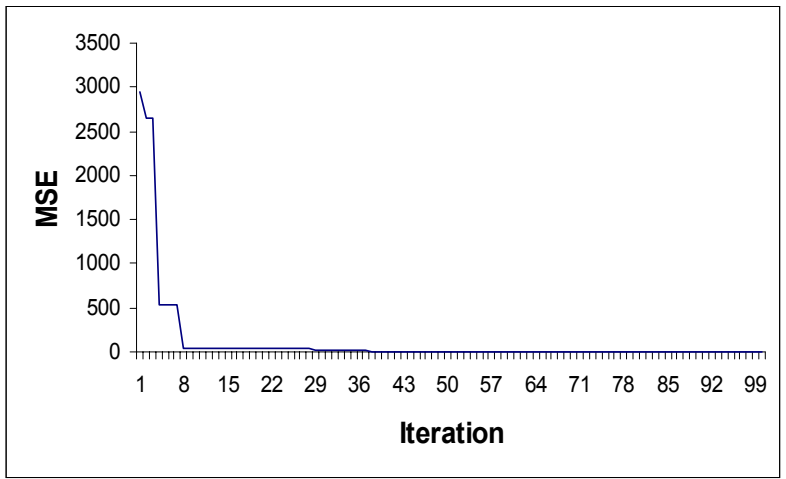

Figure 3. MSE vs. Iteration for approximating $\mathrm{Y}=\sin (\mathrm{x})$.

Based on the SIMD architecture, CLA-EC has been implemented on FPGA and some benchmark problems have been used for evaluating the performance of the algorithm. One the benchmark problems is DeJong's first function, that is

$$
F_{1}=f\left(x_{1}, x_{2}, x_{3}, x_{4}\right)=\sum_{j=1}^{4} x_{j}^{2} ;-512 \leq x_{j} \leq 512
$$

Figure 5 shows the convergence of hardware implementation that is based on the average of five different runs. Beside this experiment, CLA-EC with different numbers of cells has been implemented for optimizing $F_{1}$. Figure 6 shows the impact of this parameter on convergence of the algorithm.

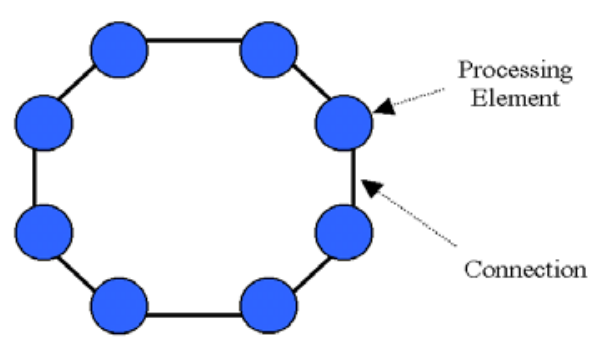

Figure 4. Ring topology

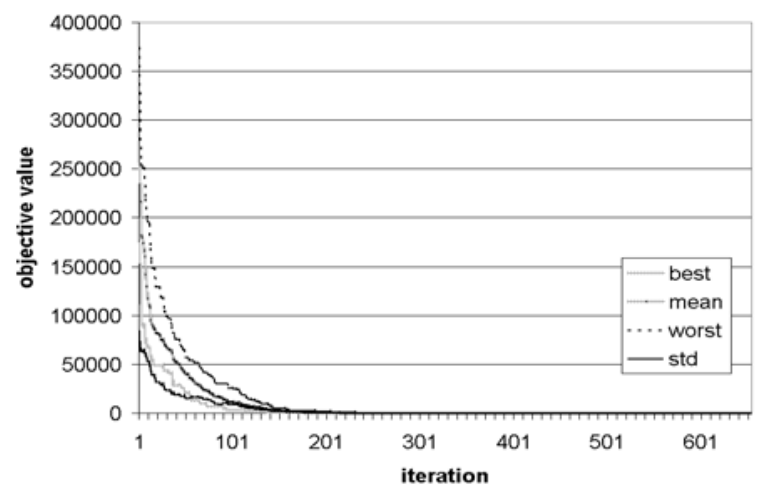

Figure 5. Convergence of $F_{1}$

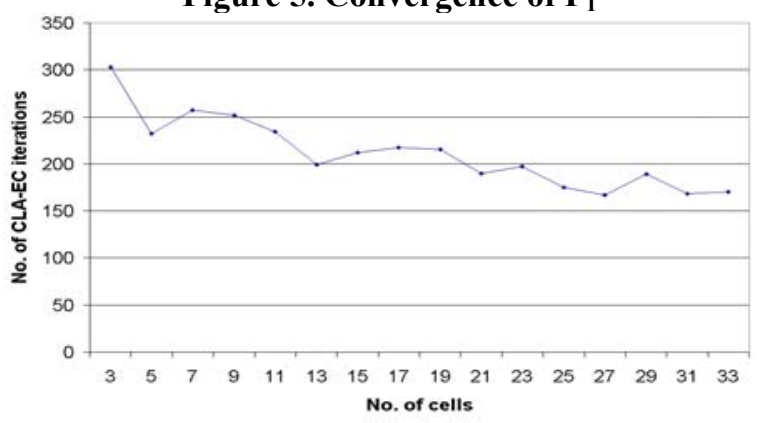

Figure 6. The effect of the number of cells on convergence for $F_{1}$

The result of the hardware synthesis of CLA-EC on Xc2v10000f957 from Virtex II family has been shown in Table 1 that includes the utilization information and clock frequency of the architecture. Consequently, this implementation provides the speed-ups of 1632.65, 3979.59 and 5612.24 for CLA-EC with 5, 10 and 15 cells respectively comparing the software implementation on Pentium IV with 256 MB RAM using C++. 
Table 1. Synthesis results

\begin{tabular}{|c|c|c|c|c|c|c|c|}
\hline & Problem Specification & \multicolumn{6}{|c|}{ Synthesis Result } \\
\cline { 2 - 7 } & $\begin{array}{c}\text { Genome } \\
\text { length }\end{array}$ & $\begin{array}{c}\text { Number } \\
\text { of Cells }\end{array}$ & FPGA & $\begin{array}{c}\text { CLB } \\
\text { Slices }\end{array}$ & $\begin{array}{c}\text { Function } \\
\text { Generators }\end{array}$ & $\begin{array}{c}\text { Dffs or } \\
\text { Latches }\end{array}$ & $\begin{array}{c}\text { Clock } \\
\text { Frequency }\end{array}$ \\
\hline $\begin{array}{c}\text { DeJong } \\
\mathrm{F}_{1}\end{array}$ & 40 bits & 5 & XC2V10000bf957 & $14.73 \%$ & $14.73 \%$ & $2.38 \%$ & $60.9 \mathrm{MHz}$ \\
\hline
\end{tabular}

\section{Conclusion}

We proposed the CLA-EC as a new algorithm for Intrinsic Hardware Evolution. CLA-EC is parallel algorithm that can benefit the hardware implementation. We conducted some experiments to evaluate its performance. In addition, SIMD based architecture was introduced to do the hardware evaluation. The results showed that the CLA-EC can be easily implemented in hardware with a considerable speed-up comparing the software implementation. As a future work, we are designing a architecture to have an evolutionary neural network on a chip that uses CLA-EC as the evolutionary algorithm.

\section{References}

[1] K. S. Narendra, and M. A. L. Thathachar, Learning Automata: An Introduction, Printice-Hall Inc, 1989.

[2] R. Rastegar and M. R. Meybodi, "A New Evolutionary Computing Model based on Cellular Learning Automata", IEEE Conference on Cybernetics and Intelligent Systems 2004 (CIS2004), Singapore, December 2004.

[3] S. Wolfram, Cellular Automata and Complexity, Perseus Books Group, 1994

[4] R. Rastegar, A. Hariri, A. Arasteh and M. R. Meybodi, "A Fuzzy Clustering Algorithm using Cellular Learning Automata based Evolutionary Algorithm", International Conference on Hybrid Intelligent Systems (HIS 2004), Japan, 2004. (Available on IEEE Computer Society)

[5] R. Rastegar, M. Rahmati and M. R. Meybodi, "A CLA-EC based Clustering Algorithm", International Conference on Adaptive and Natural Computing Algorithms, Luxemburg, October 2004.

[6] H. Beigy and M. R. Meybodi, "A Mathematical Framework for Cellular Learning Automata", Advances on Complex Systems, Vol. 7, Nos. 3-4, pp. 295-320, September/December 2004

[7] H. Beigy and M. R. Meybodi, "Open Synchronous Cellular Learning Automata", Journal of Computer Science and Engineering, Accepted for Publication. [8] N. Yoshida, T. Yasuoka, "Multi-GAP: parallel and distributed genetic algorithms in VLSI Systems", IEEE International Conference on Man, and Cybernetics, 1999.
[9] S. Scott, A. Seth, "HGA: A Hardware-Based Genetic Algorithm", In proceeding of ACM/SIGDA $3^{\text {rd }}$ International Symposium on Field Programmable Gate Arrays, 1995.

[10] J. Gallagher, S. Vigraham, G. Kramer,"A Family of Compact Genetic Algorithms for Intrinsic Evolvable Hardware", IEEE Transactions on Evolutionary Computation, Vol. 8, No. 2 April 2004

[11] K. A. De Jong, The Analysis of the behavior of a class of genetic adaptive systems, Ph.D. Dissertation, University of Michigan, Ann Arbor, 1975. 